\title{
Prebiotic effect of inulin-type fructans on faecal microbiota and short-chain fatty acids in type 2 diabetes: a randomised controlled trial
}

\author{
Eline Birkeland ${ }^{1,2}(0) \cdot$ Sedegheh Gharagozlian ${ }^{1} \cdot$ Kåre I. Birkeland $^{2,3} \cdot$ Jørgen Valeur $^{4,5} \cdot$ Ingrid Måge $^{6} \cdot$ Ida Rud $^{6}$. \\ Anne-Marie Aas ${ }^{1,2}$
}

Received: 29 January 2020 / Accepted: 11 May 2020 / Published online: 21 May 2020

(c) The Author(s) 2020

\begin{abstract}
Purpose Compared to a healthy population, the gut microbiota in type 2 diabetes presents with several unfavourable features that may impair glucose regulation. The aim of this study was to evaluate the prebiotic effect of inulin-type fructans on the faecal microbiota and short-chain fatty acids (SCFA) in patients with type 2 diabetes.

Methods The study was a placebo controlled crossover study, where 25 patients (15 men) aged 41-71 years consumed $16 \mathrm{~g}$ of inulin-type fructans (a mixture of oligofructose and inulin) and 16-g placebo (maltodextrin) for 6 weeks in randomised order. A 4-week washout separated the 6 weeks treatments. The faecal microbiota was analysed by high-throughput $16 \mathrm{~S}$ rRNA amplicon sequencing and SCFA in faeces were analysed using vacuum distillation followed by gas chromatography. Results Treatment with inulin-type fructans induced moderate changes in the faecal microbiota composition (1.5\%, $p=0.045$ ). A bifidogenic effect was most prominent, with highest positive effect on operational taxonomic units (OTUs) of Bifidobacterium adolescentis, followed by OTUs of Bacteroides. Significantly higher faecal concentrations of total SCFA, acetic acid and propionic acid were detected after prebiotic consumption compared to placebo. The prebiotic fibre had no effects on the concentration of butyric acid or on the overall microbial diversity.

Conclusion Six weeks supplementation with inulin-type fructans had a significant bifidogenic effect and induced increased concentrations of faecal SCFA, without changing faecal microbial diversity. Our findings suggest a moderate potential of inulin-type fructans to improve gut microbiota composition and to increase microbial fermentation in type 2 diabetes.

Trial registration The trial is registered at clinicaltrials.gov (NCT02569684).
\end{abstract}

Keywords Prebiotics - Type 2 diabetes $\cdot$ SCFA $\cdot$ Faecal bacteria $\cdot 16 \mathrm{~S}$ rRNA sequencing

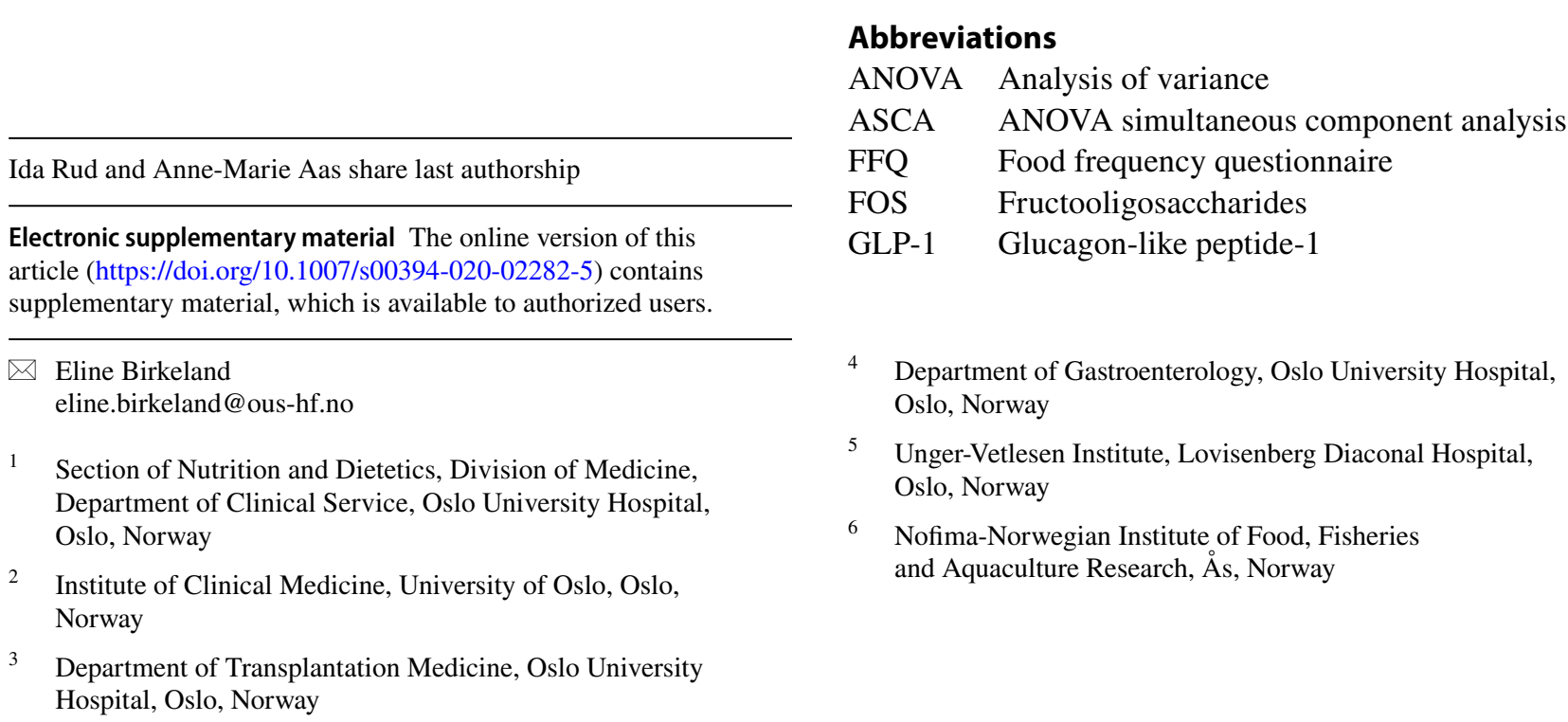




$\begin{array}{ll}\text { MCS } & \text { MiSeq Control Software } \\ \text { OUT } & \text { Operational taxonomic unit } \\ \text { PCR } & \text { Polymerase chain reaction } \\ \text { PLS-DS } & \text { Partial least squares discriminant analysis } \\ \text { PLSR } & \text { Partial least squares regression } \\ \text { PYY } & \text { Peptide YY } \\ \text { Q30 } & \text { Quality score of 30 } \\ \text { QIIME } & \text { Quantitative Insight into Microbial Ecology } \\ \text { SCFA } & \text { Short-chain fatty acids } \\ \text { VIP } & \text { Variable importance in prediction }\end{array}$

\section{Introduction}

Advice on diet and physical activity are the cornerstones of treatment of type 2 diabetes for regulation of blood glucose and prevention of long-term complications. Dietary recommendations include a diet rich in dietary fibres [1]. Dietary fibres may have several beneficial effects on glycaemic control, including slowing the rate of nutrient absorption [2,3], and modifying the gut microbiota. Prebiotic fibres evade degradation in the small intestine and are fermented into short-chain fatty acids (SCFA) in the colon by presumed health promoting gut bacteria, stimulating their growth and activity [4]. The wide-ranging health benefits of bifidobacteria in particular, are well documented [5].

The SCFA produced by gut bacteria, mainly the acetic, propionic and butyric acids, are used as an energy source for the colonocytes and substrates for the hepato-metabolic pathways $[6,7]$. The SCFA may also act as signalling molecules by binding to receptors on the enteroendocrine cells, with the potential to increase postprandial secretion of gut hormones and improve regulation of blood glucose [7]. Thus, increased production of SCFA, especially butyric acid, is considered favourable [8-10].

Observational studies have shown that gut microbiota in type 2 diabetes differs from healthy individuals with lower diversity of the microbial community, less of the butyrateproducing bacteria, and lower faecal concentrations of SCFA [11-13]. Elevated levels of pathogenic bacteria, and functions related to oxidative stress response, such as enrichment of catalase and increased production of the antioxidant glutathione were also found [12]. Alterations in gut homeostasis such as these are suspected to contribute to the pathophysiology of type 2 diabetes [7].

Improvement of the microbial profile in the gut could benefit individuals with type 2 diabetes in particular, by enhancing the production of SCFA. The inulin-type fructans and galactooligosaccharides are the most studied prebiotic fibres and inulin-type fructans are also extensively used as industrial food ingredients. Numerous trials show that inulin-type fructans supplemented in doses varying between 5 and $30 \mathrm{~g}$ per day may increase the abundance of bifidobacteria and
SCFA in faeces, and enrich microbial diversity in healthy people and in non-diabetic patients [14-24]. Interestingly, lower levels of bifidobacteria have been reported in individuals with type 2 diabetes compared to healthy individuals, and probiotic supplementation with this genus has been reported to improve glucose tolerance in animal studies [25]. Furthermore, studies conducted in type 2 diabetes patients have also shown that dietary fibres with and without prebiotic abilities could improve glucose metabolism [2, 26]. Yet, to the best of our knowledge, it has not been investigated whether inulin-type fructans have different impact on gut microbiota and fermentation in people with diabetes than in healthy individuals.

The aim of this study was, thus, to evaluate the prebiotic effect of inulin-type fructans on faecal microbiota and SCFA in patients with type 2 diabetes. We hypothesised that treatment with inulin-type fructans for 6 weeks would induce positive changes in the composition of gut microbiota, such as enriching concentrations of bifidobacteria and butyrate producers, increasing the microbial diversity, and increasing concentrations of faecal SCFA.

\section{Methods}

\section{Trial design}

We conducted a randomised, placebo controlled and doubleblind crossover trial between February 2016 and December 2017 at the Diabetes Research laboratory, Oslo University Hospital, Aker. Due to high inter-individual variability in the microbial response to dietary interventions, the crossover approach was selected over a parallel design, allowing the participants to serve as their own controls. This study is part of a trial where the primary aim was to investigate the effect of prebiotics on GLP-1 response. These results are not yet published. The trial was approved by the Regional Ethics Committee for Medical and Health Research and registered at clinicaltrials.gov (NCT02569684). Written informed consent was obtained from all patients prior to their inclusion in the study. The study was performed in accordance with the ethical standards laid down in the 1964 Declaration of Helsinki and its later amendments.

\section{Participants}

Adult men and women with type 2 diabetes were invited consecutively as they attended the Diabetes Outpatient Clinic. Participants were also recruited from advertisement in social media, the hospital lobby and pharmacies, and from general practices.

Eligibility for participation was determined at a screening visit at least 4 weeks prior to enrollment. Eligible 
patients had a BMI $\leq 40 \mathrm{~kg} / \mathrm{m}^{2}, \mathrm{HbA}_{1 \mathrm{c}}<10.0 \%(86 \mathrm{mmol} /$ $\mathrm{mol}$ ), and were not treated with insulin or glucagon-like peptide-1 (GLP-1) analogues. Exclusion criteria were fibre intake $>30 \mathrm{~g}$ per day, performance of high-intensity exercise, weight changes of $>3 \mathrm{~kg}$ within the last month, planned or present pregnancy, drug or alcohol dependence, treatment with antibiotics within the last 3 months, long distance from home to the study centre, and consumption of dietary supplements containing prebiotics or probiotics. At screening, the fibre intake was assessed based on a simplified approach where we asked the potential participants how often they consumed food items known to be important sources of fibre in the Norwegian diet, and their portion sizes. Patients diagnosed with either dementia, organic or functional gastrointestinal diseases, or had cancer within the last 5 years were not included.

In total, 131 patients were assessed for eligibility and 35 were randomised to start with either inulin-type fructans or placebo, of whom 25 completed the intervention (Online Resource 1). Of the ten patients who were randomised, but did not start or complete the intervention, no individuals were excluded or withdrew because of side effects from the supplements or other study-related procedures. One participant was excluded in the faecal microbiota analysis due to one sample with low amounts of extracted DNA.

\section{Dietary intervention}

For two periods of 6 weeks separated by a 4-week washout, the participants consumed $16 \mathrm{~g}$ per day of inulin-type fructans (a 50/50 mixture of oligofructose and inulin; Orafti ${ }^{\circledR}$ Synergy1, Beneo GmbH, Germany) and placebo (maltodextrin $16 \mathrm{~g}$ per day) in addition to their ordinary diet and in a randomised order. The dose of $16 \mathrm{~g}$ was decided after considering the amounts of prebiotics sufficient to induce positive and significant changes in gut microbiota and GLP-1 response against doses low enough to avoid adverse side effects and minimise gastrointestinal discomfort. Trials with healthy adults have demonstrated significant increases in bifidobacteria with doses of inulin-type fructans from $5 \mathrm{~g}$ per day $[14,27]$ and that $10 \mathrm{~g}$ per day is preferred rather than $20 \mathrm{~g}$ when also taking side effects into consideration [27]. Furthermore, Cani et al. demonstrated that 16-g inulin-type fructans per day induced increased response of GLP-1, and only minor gastrointestinal symptoms in healthy adults [28]. The supplements were powdered, similar in colour and taste, and were wrapped in identical and non-transparent portion packages of $8 \mathrm{~g}$. For adaptation, the participants consumed only $8 \mathrm{~g}$ per day during the first week and progressed to $16 \mathrm{~g}$ per day for the remaining 5 weeks. The participants added the supplements to food or drinks and consumed it whenever they preferred. They returned unused supplement packages, and the number of unused sachets was used as an estimate of compliance.

\section{Outcomes and data collection}

Before and after the 6-week intervention periods, the participants attended the hospital for visits, where they delivered faecal samples for analysis of microbiota and SCFA. For a comprehensive assessment of diet, the participants filled out food frequency questionnaires (FFQ) before the first intervention period. The participants were instructed to avoid making changes in habitual lifestyle during the trial and to avoid strenuous exercise one day in advance of the visits. They were also told not to make any changes regarding medication during the study and to discontinue diabetes medication two days prior to the visits.

\section{Anthropometric measurements}

Weight and bioimpedance were measured using a body composition analyser (Tanita BC-418 MA Segmental Body Composition Analyzer) at the four visits, before and after the intervention periods. Height was measured with a standard altimeter. Participants were examined with bare feet wearing light clothing.

\section{Assessment of diet}

The FFQ is a validated, self-administered, paper-based optical mark readable questionnaire assessing the total diet [29, 30]. Participants were instructed to fill in questionnaires based on eating habits during the last 6 weeks.

\section{Faecal collection}

The participants were provided with sterile plastic containers to collect faecal samples at home, and instructed to store these instantly in a freezer one day prior to each of the four visits. The samples were brought to the clinic in cooler bags containing freezer blocks and immediately stored at $-80{ }^{\circ} \mathrm{C}$ for later analysis.

\section{Microbiota analysis}

\section{DNA extraction and microbiota analysis}

Bacterial DNA was extracted from faecal content (approximately $100 \mathrm{mg}$ ) by mechanical and chemical lysis using the DNaeasy PowerSoil HTP 96 Kit (Qiagen), following the manufacture's protocol. The mechanical lysis step with bead beating was done twice using the FastPrep ${ }^{\circledR}-96$ homogenizer (MP Biomedicals) for $60 \mathrm{~s}$ at $1600 \mathrm{rpm}$. Then, samples were centrifuged for $6 \mathrm{~min}$ at $4500 \times g$ as described in 
the protocol. The microbiota was analysed by $16 \mathrm{~S}$ rRNA amplicon sequencing $(2 \times 150 \mathrm{bp})$ of the variable region 4 following an in-house protocol [31], which is presented in detail in supplementary methods of Caporaso et al. [32]. The current primers [33-35] have been modified from the original 515F-806R primer pair, with barcodes now on the forward primer and degeneracy added to both the forward and reverse primers to remove known biases. The sequencing was done on a MiSeq (Illumina) at Nofima using pooled polymerase chain reaction (PCR) samples, which were based on triplicate PCRs per DNA sample using sample-specific barcoded forward primers. PhiX Control v3 was included and accounted for $10 \%$ of the reads. The MiSeq Control Software (MCS) version used was RTA 1.18.54.

\section{Data processing of sequencing data}

Data processing of the sequencing reads was performed using the pipelines in Quantitative Insight Into Microbial Ecology (QIIME) v.1.9 [36]. Briefly, the total number of reads was $15,217,265$ followed by $9,007,278$ reads after joining forward and reverse reads and removal of barcodes that failed to assemble. The sequences were demultiplexed into representative sample taqs and quality filtered, allowing zero barcode errors and a quality score of 30 (Q30), resulting in 7,550,212 sequences. Reads were assigned to their respective bacterial taxonomy (operational taxonomic unit: OTU) by clustering them against the Greengenes reference sequence collection (gg_13_8) using a 97\% similarity threshold. Reads that did not hit a sequence in the reference sequence collection were clustered de novo. Chimeric sequences were removed using ChimeraSlayer, and all OTUs that were observed fewer than 2 times were discarded. This resulted in an OTU table containing 15,168 different OTUs, which was based on a total of $6,642,085$ read counts. The OTU table was used for microbial (alpha) diversity analysis using equal number of sequences across samples, i.e. alpha rarefaction, where the OTU table was resampled to an even depth of 13,000 sequences per sample. Summary tables at phylum, order, family and genus levels were constructed from the OTU table (i.e. OTU level/species level). The data were transformed by centred $\log 2$ ratios, to stabilize the variation and remove dependencies between abundance variables. At any taxonomic level, bacteria groups that were present in less than $50 \%$ of the subjects were combined into one group (called "rare"), as it is not possible to make statistical inference on individual rare bacteria groups. Square brackets around taxonomic names (e.g. [Ruminococcus]) are taxa proposed by Greengenes based on genomic trees, but are not verified taxonomies.

\section{SCFA analysis}

Upon analysis, $0.5 \mathrm{~g}$ of the faecal material was homogenised after addition of distilled water containing $3 \mathrm{mmol} / \mathrm{L}$ of 2-ethylbutyric acid (as internal standard) and $0.5 \mathrm{mmol} / \mathrm{L}$ of $\mathrm{H}_{2} \mathrm{SO}_{4} ; 2.5 \mathrm{~mL}$ of the homogenate was vacuum distilled, according to the method of Zijlstra et al. [37], as modified by Høverstad et al. [38]. The distillate was analysed with gas chromatography (Agilent 7890 A, CA, USA), using a capillary column (serial no. USE400345H, Agilent J\&W GC columns, CA, USA), and quantified using internal standardisation. Flame ionisation detection was employed. The following SCFA were analysed: acetic, propionic, butyric, isobutyric, valeric, isovaleric, caproic and isocaproic acids. The results were expressed in $\mathrm{mmol} / \mathrm{kg}$ wet weight. In addition, we calculated the proportional distribution of individual SCFA to total SCFA.

\section{Gastrointestinal symptoms}

After both interventions the participants completed a questionnaire about changes in gastrointestinal symptoms concerning the last 6 weeks (abdominal discomfort, diarrhoea, constipation, bloating, and flatulence) with a word rating scale: much worse, worse, unchanged, better, and much better.

\section{Sample size}

The sample size was calculated based on the expected effects on the primary outcome measurement from the main study, which was change in GLP-1-response to a standardised meal. This estimation was based on results from a drug trial in patients with type 2 diabetes, where changes in GLP-1 response were the primary endpoint [39]. This provided a tentative sample size of 23 patients to achieve $80 \%$ power at alpha $=0.05$. To account for drop-outs and a possible lower treatment effect due to differences in intervention and design, we added 12 patients, giving a total of 36 patients required for randomisation.

\section{Randomisation and blinding}

Staff not involved in the study performed subject randomisation and product distribution. Randomisation lists were generated using a randomisation command for two by two cross-over studies in Stata 14. All participants and clinical researchers were blinded to treatment allocation and the randomisation key was not broken before all data were collected, the database was washed and the laboratory analyses were performed. 


\section{Statistical analyses}

SPSS version 25.0 software was used for descriptive statistics and analyses of biochemical responses. Baseline characteristics are reported as mean (range), (SD) or $n(\%)$. The variables, total SCFA as well as the individual SCFA, were skewed and their distribution did not improve with $\log$ transformation. The effects of inulin-type fructans on SCFA were, thus, analysed using Wilcoxon Signed Rank test and $P<0.05$ (two tailed) was considered as statistically significant. The results from SCFA analyses are reported as medians (25th-75th percentiles).

The observed variation in microbiota at different taxonomic levels were decomposed by analysis of variance (ANOVA) simultaneous component analysis (ASCA) [40]. The carry-over effect was originally included in the model by the effects Period + Treatment $\times$ Week $\times$ Period, but were removed as they were non-significant. The final ASCA model contained a Subject effect, accounting for the between subjects variation, and a intervention-specific Treatment $\times$ Week effect. Post hoc comparisons between factor levels of the intervention design were performed using partial least squares discriminant analysis (PLS-DA) after removing the between-subjects variation [41]. Bacteria that discriminate the prebiotic fibres from placebo and baseline levels were identified by variable importance in prediction (VIP) combined with Pearson correlations between individual bacteria's group means and class labels [41]. A cutoff of 1.2 was used for VIP and 0.9 for correlation. Effect sizes were calculated as difference between means after prebiotic treatment compared to placebo treatment and baseline values combined.

The microbial diversity, represented by the metrics Observed OTUs, Phylogenetic Distance (PD) whole tree and Chao1, was analysed using a Mixed-Effects Model in Minitab ${ }^{\circledR}$ 18.1. Treatment, Week and Period were defined as fixed effects and Subject as random.

Partial least squares regression (PLSR) was used to analyse the relationship between microbiota (OTU level) and the different SCFA/metformin users (yes or no) without taking the intervention into account and validated by crossvalidation [41]. Variable importance was estimated by the VIP method. Individual variation in effect size of the intervention (subject-specific effect sizes) on the Bifidobacterium genus and its OTUs were used to relate against baseline data, i.e. initial level of Bifidobacterium, microbial diversity and fibre intake ( $\mathrm{g} /$ day) characteristics. Data of none identified relationships (i.e. metformin, Bifidobacterium, microbial diversity and fibre intake) are not presented. The multivariate statistical analyses were performed using MATLAB (R2018b, The MathWorks Inc.).
Table 1 Baseline characteristics of study participants

\begin{tabular}{|c|c|}
\hline & $(n=25)$ \\
\hline Women & $10(40.0)$ \\
\hline Age (years) & $63.1(41-73)$ \\
\hline Fasting glucose $(\mathrm{mmol} / \mathrm{L})$ & $8.7(4.0-12.8)$ \\
\hline BMI $\left(\mathrm{kg} / \mathrm{m}^{2}\right)$ & $29.1(19-39)$ \\
\hline $\mathrm{HbA} 1 \mathrm{C}(\%)$ & $6.9(5.1-9.6)$ \\
\hline$(\mathrm{mmol} / \mathrm{mol})$ & $51.9(32.2-81.4)$ \\
\hline Energy (kcal/day) & $2338(1315-4658)$ \\
\hline Proteins (E\%) & $18.1(9.6-22.9)$ \\
\hline Fat $(\mathrm{E} \%)$ & $36.9(21.7-44.7)$ \\
\hline Carbohydrates (E\%) & $38.9(27.4-60.3)$ \\
\hline Dietary fibre (g/day) & $32.2(9.6-54.7)$ \\
\hline Diabetes duration (years) & $4.7(0.2-20.0)$ \\
\hline \multicolumn{2}{|l|}{ Diabetes treatment } \\
\hline Diet & $8(32.0)$ \\
\hline Metformin & $17(68.0)$ \\
\hline SLGT2 inhibitors $^{\mathrm{a}}$ & $2(8.0)$ \\
\hline DPP-4 inhibitors ${ }^{\mathrm{a}}$ & $5(20.0)$ \\
\hline Sulfonylureas ${ }^{\mathrm{a}}$ & $1(4.0)$ \\
\hline Proton pump inhibitors & $0(0)$ \\
\hline
\end{tabular}

Values are mean (range) or $n(\%)$

${ }^{\mathrm{a}}$ Medication used in addition to Metformin

\section{Results}

\section{Patient characteristics}

Baseline characteristics of the 25 participants who completed the intervention are presented in Table 1 . Forty percent were women, the overall mean age was 63.1 years, BMI $29.1 \mathrm{~kg} / \mathrm{m}^{2}, \mathrm{HbA}_{1 \mathrm{C}} 6.9 \%$ [52 $\left.\mathrm{mmol} / \mathrm{mol}\right]$, and diabetes duration was 4.7 years. Two thirds of participants received glucose lowering medications. The intake of dietary fibre assessed with FFQ at the first visit (baseline) turned out to be higher than expected, as the evaluation of fibre intake at the screening was based on a simpler approach with questioning about how often a few certain food items were consumed and their portion sizes. Apart from a reported higher intake of dietary fibre (mean $32.2 \pm 10.3 \mathrm{~g} /$ day), the participants characteristics seemed to be representative of patients with type 2 diabetes in Norway.

The compliance was excellent with mean (range) 96.7 (79.2-100.0)\% of the prebiotic supplement and 95.7 (77.9-100.0)\% of the placebo consumed.

Individual faecal microbiota and effects of inulin-type fructans.

The faecal microbiota was analysed from 24 participants who completed the two crossover periods with four sampling times per individual. Statistical overview of the microbiota data is presented in the online supporting material (Online 
Resources 2, 4 and 5), also confirming no differences in microbiota composition nor microbial diversity between crossover periods.

The microbiota data show abundant inter-individual variability of microbiota composition, (explaining $>60 \%$ of total variation) and minor effect of the prebiotic fibre (explaining $<2.5 \%$ ) (Online Resource 2). Overview of the inter-individual variation of phyla at baseline is presented in Fig. 1, showing the gradient distribution of the dominating Bacteroidetes (mean abundance of $69 \%$ ), with a trade-off with Firmicutes (26\%) as the second dominating phylum. Indeed, except for two participants, Bacteriodetes accounted for more than $50 \%$ of the microbiota present in the individuals. Tenericutes (1.5\%), Proteobacteria (1.2\%), Actinobacteria $(0.9 \%)$, Verrucomicrobiota $(0.7 \%)$ and Cyanobacteria $(0.3 \%)$ were also present to a variable degree between individuals.

The moderate changes in microbiota composition after intervention with the prebiotic fibre were explained by only $2.2 \%$ and $1.5 \%$ of the total variation at phylum and OTU (species) levels, respectively (Online Resource 2). The overall microbiota effect did not reach significance at the phylum level $(p=0.091)$, although Actinobacteria (VIP 1.32) was significantly positively affected by prebiotic fibre compared to placebo and baselines after the 6 weeks of intervention (Online Resource 3). However, the prebiotic fibre had significant effect at the OTU level $(p=0.045)$, with significant impact on 32 OTUs (Online Resource 4). These are presented in Fig. 2, with their representative effect sizes.

Indeed, the three OTUs with highest positive effect sizes were of Actinobacteria and assigned to Bifidobacterium adolescentis. Bifidobacterium adolescentis OTU559527 was the most abundant of these OTUs $(0.6 \%)$. The remaining OTUs positively related to prebiotic fibre intake were not that highly ranked and with less effect sizes, and were mostly of Bacteroidetes origin or Firmicutes. Especially, OTUs within Bacteroides were among these, including one dominating OTU assigned to Bacteroides ovatus, and three OTUs within Clostridiales, including Lachnospiraceae and Faecalibacterium prausnitzii. The OTUs that decreased with the prebiotic fibre were of Firmicutes, including dominating OTUs assigned to the families Ruminococcaceae (Ruminococcus) and Lachnospiraceae ([Ruminococcus]), all with high effect size. In addition, an OTU of Erysipelotrichaceae declined with the prebiotic fibre.

Microbial diversity was not affected by the prebiotic fibre after the 6-week intervention (Online Resource 5), as exemplified with the metrics observed OTUs (Fig. 3).

\section{Effects of inulin-type fructans on faecal SCFA}

The intervention resulted in a significant increase in faecal concentrations of total SCFA $(p=0.04)$, acetic acid $(p=0.02)$, and propionic acid $(p=0.04)$ as compared to placebo (Table 2). There was no difference in effect on butyric acid between the treatments $(p=0.19)$.

\section{Relationship between microbiota and SCFA}

The relationship between microbiota and the SCFA (acetic, propionic, butyric and valeric acid) is presented in a heatmap, only including the OTUs significantly affected by the prebiotic intervention (Fig. 4). A general trend was that acetic acid was positively related to OTUs that increased with the prebiotic fibre. The opposite trend was observed for the OTUs that declined with the prebiotic treatment. Interestingly, the prebiotic affected OTUs of Bifidobacterium adolescentis were negatively related towards butyric acid. Only Lachnospiraceae OTU514272 was positively related to butyric acid among the prebiotic affected OTUs. Another trend was that valeric acid was positively related to the OTUs that declined with the prebiotic fibre.
Fig. 1 Relative abundance (\%) of the dominating phyla in faeces of the participants at baseline

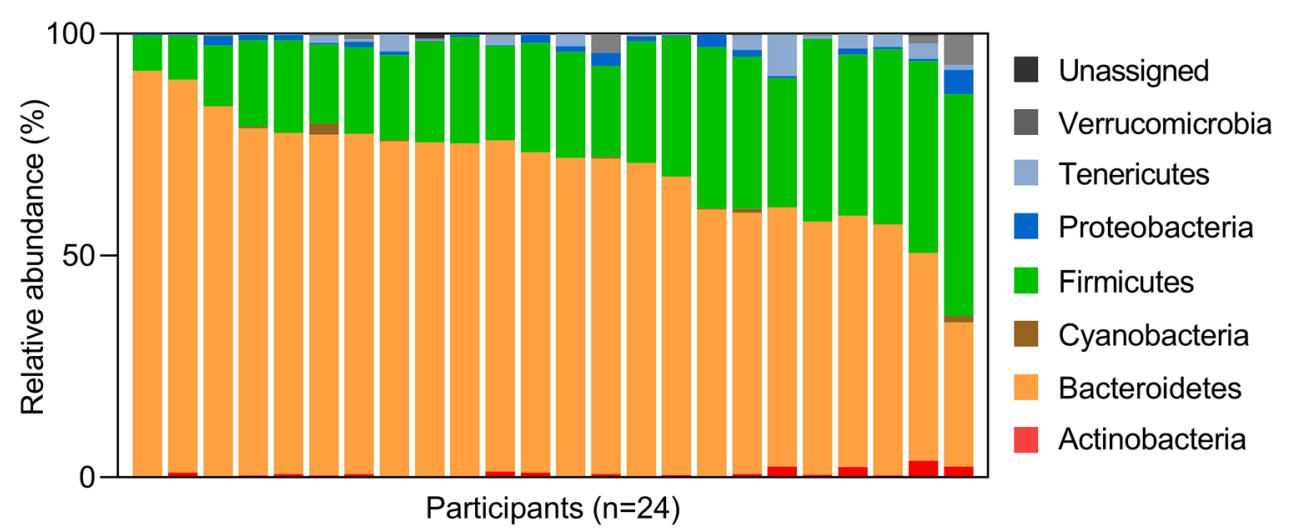



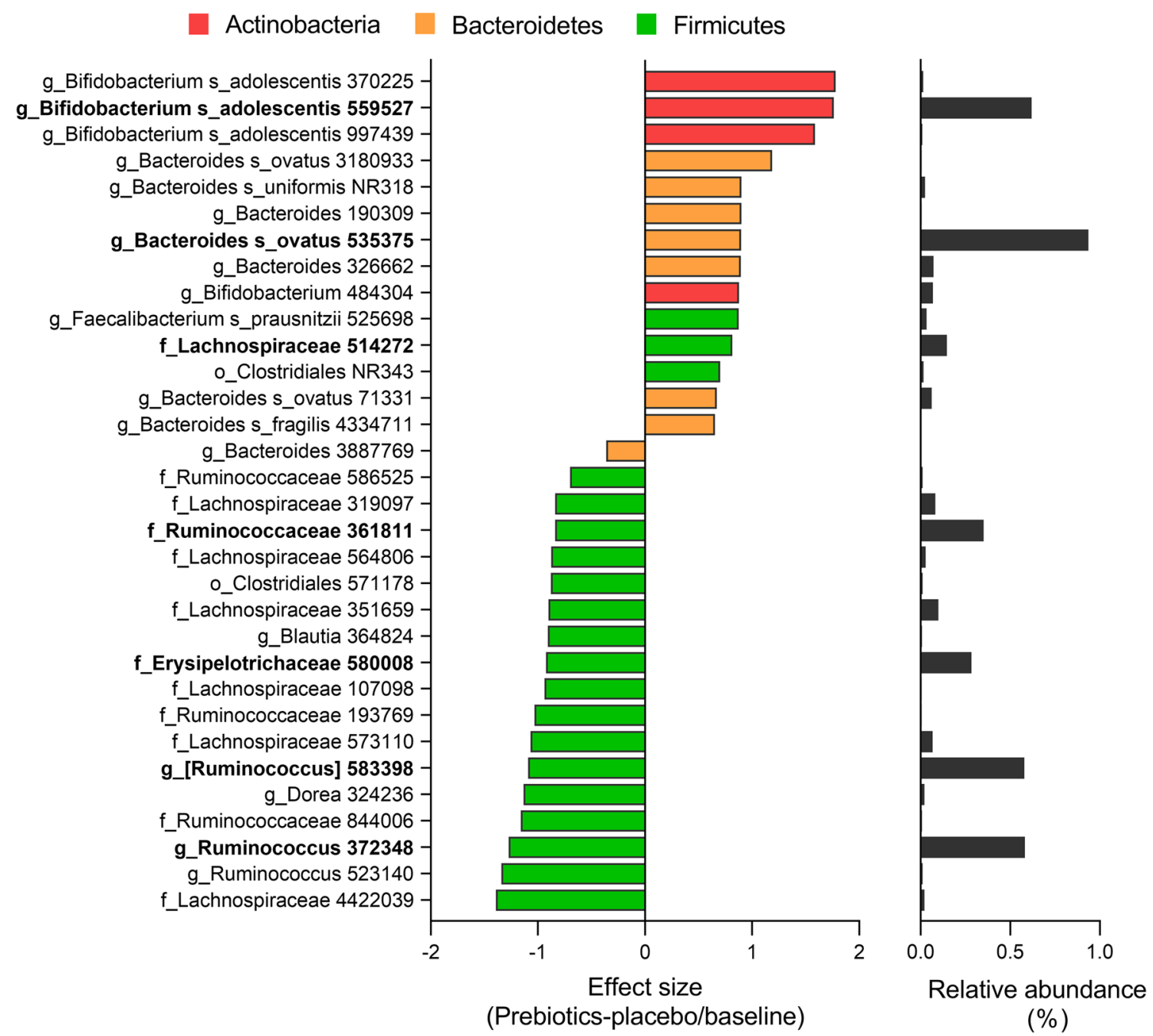

(Prebiotics-placebo/baseline)

(\%)

Fig. 2 OTUs affected by the prebiotic intervention for 6 weeks sorted by effect size. Effect size is the differences between prebiotic intervention period compared to placebo period /baseline (log2). Dominating OTUs $(>0.1 \%)$ are indicated in bold, and the relative average

\section{Discussion}

In this randomised controlled trial in patients with type 2 diabetes, we found that $16 \mathrm{~g}$ per day of a 50/50 mixture of inulin and oligofructose supplemented for 6 weeks caused an increase in bifidobacteria and SCFA in faeces, compared to maltodextrin. However, the prebiotic fibre had no effect on butyric acid or the overall microbial diversity. To the best of our knowledge, this is the first trial studying the effect of inulin-type fructans on faecal microbiota and SCFA in people with type 2 diabetes.

In planning the present trial, we decided on inulin-type fructans as choice of prebiotic fibres. These are the most studied among prebiotics and a mixture of both longand short-chain inulin have been proposed to minimise the expected gastrointestinal symptoms [42, 43]. The abundance of the OTUs is included at the right. Brackets indicate candidate taxonomy. Bars are coloured according to representative phylum

bifidogenic effect found in the present trial is in accordance with other human studies with doses of inulin-type fructans varying between 5 and 30 g per day, in healthy people and in non-diabetic patients [14-24]. We thus belive a dose of $16 \mathrm{~g}$ per day to be sufficient. However, the prebiotic effect on microbiota composition in the present study was moderate, accounting for only a few percentage of variation in the microbiota. This has also been demonstrated in healthy humans given inulin as prebiotic [22], and may be explained by the large individual variation in microbiota between the participants.

Although bifidobacteria are unable to produce butyric acid themselves, they are valuable in cross-feeding where various species metabolise non-digestible carbohydrates through several steps. The bifidobacteria contribute with their ability to degrade fructan chains and, thus, prepare 


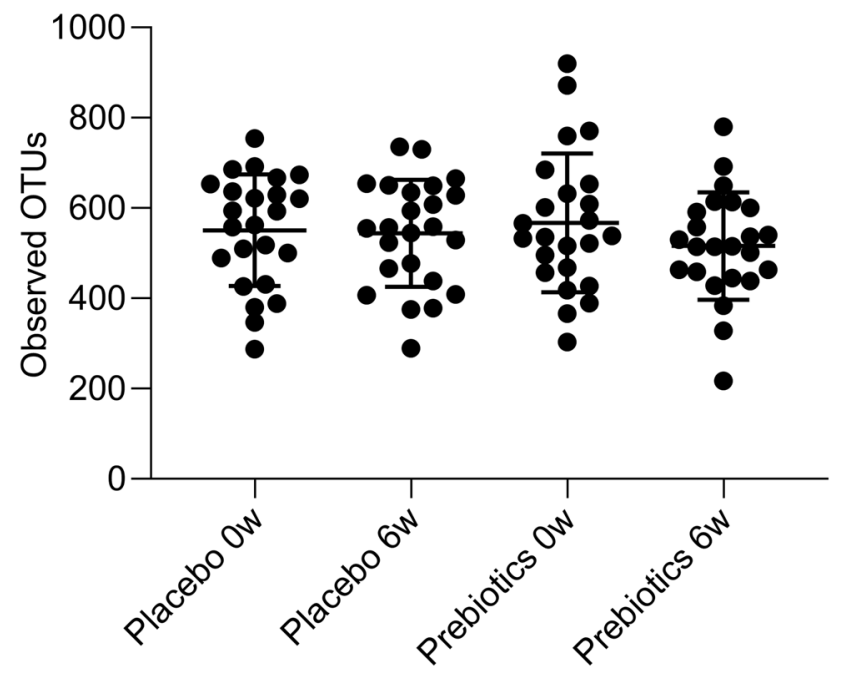

Fig. 3 Microbial diversity shown as number of observed OTUs between prebiotics and placebo at baseline $(0 \mathrm{w})$ and after treatment period of 6 weeks $(6 w)$

for other species to complete the fermentation [19]. The extensive health benefits of bifidobacteria are well documented [5]. Studies also confirm bifidogenic health benefits of particular interest in type 2 diabetes [44, 45]. Apart from anti-carcinogenic properties and positive effects on blood lipids, trials in humans and mice report that bifidobacteria also may prevent endotoxemia and improve regulation of blood glucose [44-46].

The prebiotic treatment did not have the desired effect of increased microbial diversity in our participants. Tandon et al. found increased diversity of faecal bacteria in a healthy population after supplementing fructooligosaccharides (FOS) [47], but others found no or even decreased effect of inulin-type fructans $[19,21,22]$. These studies were all conducted in healthy adults, but with varying treatment doses and degrees of polymerization. The trial performed by Tandon et al. however, stands out with a particular long treatment duration (3 months) and lower treatment dose. This may indicate that it takes longer to affect the microbial diversity than to enhance the abundance of bifidobacteria in the gut when supplementing inulin-type fructans. We chose to limit the duration of the intervention period to 6 weeks to avoid a prebiotic effect of weight loss previously reported $[48,49]$, as weight loss could potentially have confounded other outcome measures.

Even though the effect of prebiotic fibre on the microbiota composition was moderate, enhanced faecal concentrations of SCFA was detected, indicating changed microbial metabolic activity in the gut. Total SCFA, acetic acid and propionic acid increased significantly. This contrasts the findings in the majority of other clinical trials that measured faecal SCFA after supplementing inulin-type fructans. Only Baxter et al. found increased concentrations of total SCFA in faecal samples from healthy individuals supplemented with $20 \mathrm{-g}$ inulin per day for 2 weeks, despite no changes in acetic or propionic acid, separately [24]. Others found no or even decreased concentrations of faecal SCFA in healthy adults with normal or overweight after treatment with 5-16-g inulin-type fructans per day for durations between 2 and 12 weeks $[18,19,21,23,24]$. Acetic and propionic acid have been linked to mechanisms preserving or improving glucose homeostasis and appear to be anti-carcinogenic, and propionic acid is able to reduce visceral and liver fat [50]. Butyric acid is of particular interest in type 2 diabetes as animal studies report it improves glucose homeostasis by inducing gut production of GLP-1 and peptide YY (PYY) [9] as well as protecting the gut barrier function [51]. However, no significant increase in faecal concentration of butyric acid was detected in the present study. This is in line with the previously mentioned human trials with inulin-type fructans showing no change or even decrease in faecal butyric acid in healthy individuals [19-21]. It is worth noticing that there was a large variability in the measured change in all SCFA, which may be due to individual differences in baseline microbiota, diet and absorption. This can also explain some of the inconsistent findings between studies.

The bifidogenic effect in the present study was related to increase in OTUs assigned to $B$. adolescentis, which were negatively related to butyric acid. Stimulation of $B$. adolescentis is in agreement with other studies using oligofructose and inulin as substrates [24, 52-55]. Fermentability of both the short- and long-chain fructans may have been an advantage of $B$. adolescentis, a capacity shown to be species- and strain dependent among the bifidobacteria [55]. However, bacterial metabolic activity reported in strictly controlled in vitro studies may not occur in the less predictable environment associated with in vivo studies.

Species of Bacteroides, e.g. B. ovatus, were also enriched by the prebiotic fibre. This genus is known for its genomic capacity to ferment a wide range of polysaccharides into acetic and propionic acid. Capability to ferment both FOS and inulin has previously been shown for $B$. ovatus both genetically and physiologically [56]. It could be speculated that the observed increase in Bacteroides species was enhanced by Bacteroides being the dominating genus among the type 2 diabetes patients. The butyrate-producing $F$. prausnitzii has in some human studies also been shown to be stimulated by intake of inulin-type fructans $[19,24,47]$. Indeed, $F$. prausnitzii was slightly enriched in this study, as well as an OTU of Lachnospiraceae that was also positively related to butyric acid. Still, the increase did not significantly affect the levels of faecal butyric acid. Low levels of butyrateproducing taxas are a well known feature of the type 2 diabetes gut and this may also explain why we did not see significant increase in faecal concentration of butyric acid 


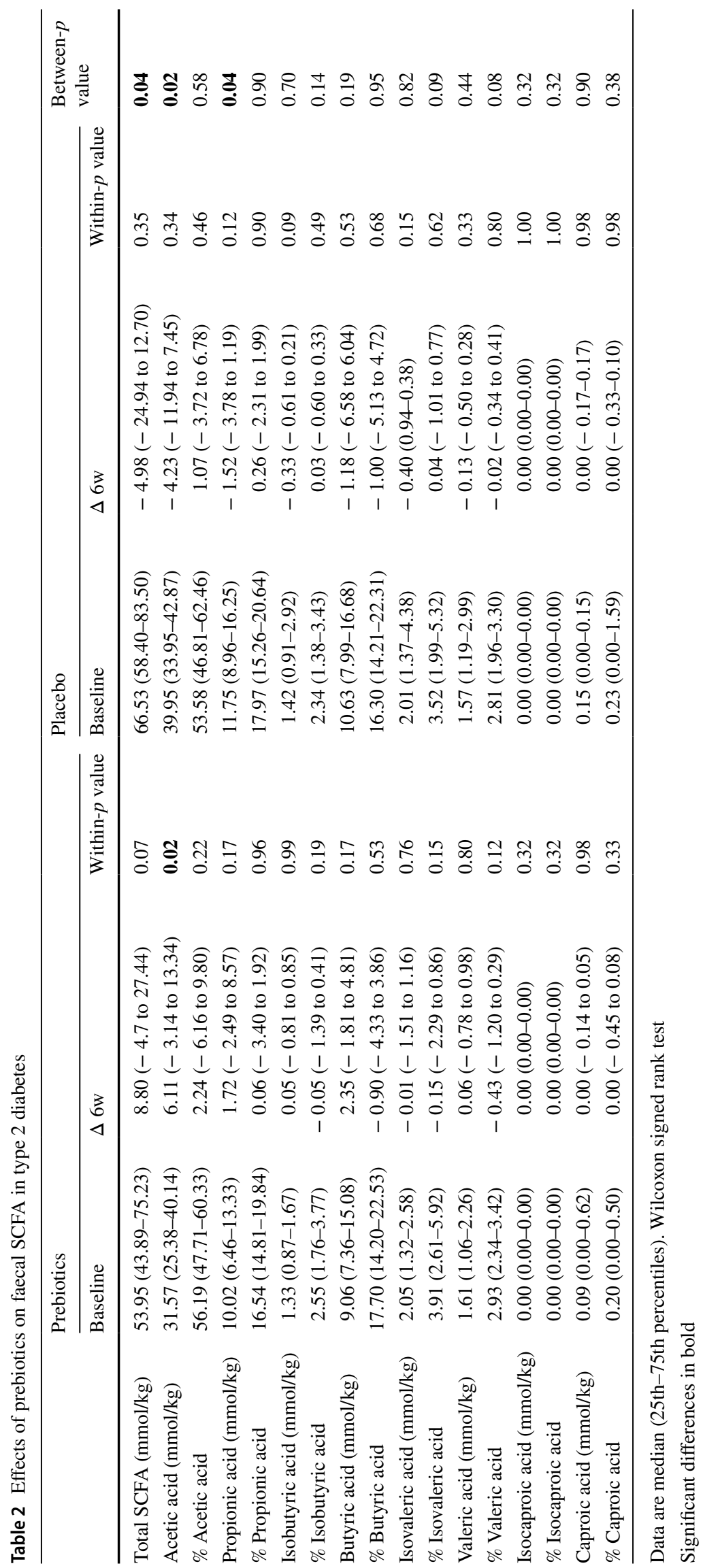


Fig. 4 Heatmap of OTUs related to SCFA by PLS regression. Only OTUs affected by the prebiotic intervention are presented and sorted by their effect sizes (as in Fig. 2). Correlation is estimated with Spearman's rho coefficient, where red is a positive and blue is a negative relation. Asterisk indicates significant relationship (VIP $>1.2$ ). Dominating OTUs $(>0.1 \%)$ are indicated in bold

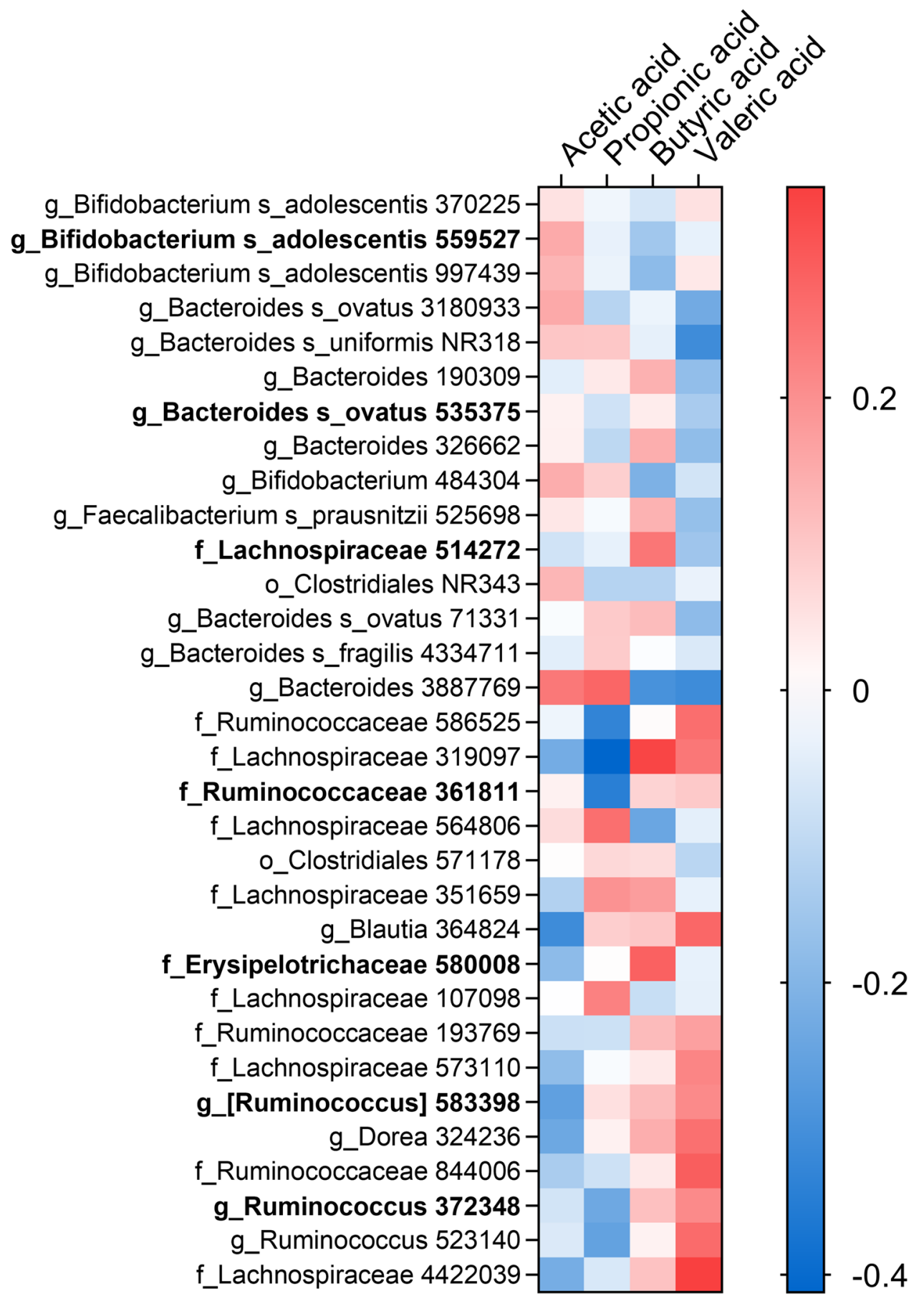

$[11,12]$. Importantly, the faecal concentrations of SCFA is only an estimate of colonic SCFA production. Inulin is rapidly fermented in the proximal colon and most of the SCFA produced are absorbed during transit through the colon, and only few percents remain in the faeces [50]. Apart from the substrate availability, SCFA concentrations in faecal samples are also determined by the absorption rate into the systemic circulation and portal vein, transit time through colon and cross-feeding establishments in the microbiota. Changed faecal SCFA is rather an indication of changed bacterial activity in the gut and thus a valuable measurement when exploring the effect of prebiotic supplements.

Lately, metformin has been shown to affect the gut microbiota, and may, thus, confound the results in clinical trials investigating the composition of gut bacteria in populations with type 2 diabetes [11]. The majority of the participants in our study (68\%) used metformin during the intervention, all with a dose that was kept unchanged, and we found no difference in the overall faecal microbiota between participants using metformin or not. 
The strengths of this study include the randomised double-blind crossover design, high level of compliance, no dropouts related to the intervention, and assessment of habitual diet and medication known as possible confounders. To minimise the risk of carry-over effects, we included a washout period of 4 weeks. The bacterial response in the gut to dietary intervention occurs within few days and returns to its original state at the same rate when the intervention is discontinued [57]. A remaining effect of prebiotics on faecal SCFA after a 4-week-long washout is, thus, unlikely and no differences between baseline concentrations before and after the washout were found (Online Resource 6).

One clear limitation of this study is measuring of faecal SCFA as a proxy for the colonic production of SCFA. The treatment duration of 6 weeks may also have been too short to enhance the microbial diversity. Another limitation is that the sample size was calculated based on expected effects on the primary outcome measurement from the main study (GLP-1 response) and not on expected effects on composition of the microbiota. However, bifidogenic effect on gut bacteria has been found in comparable studies that have evaluated the effects of inulin-type fructans, both with similar and lower sample sizes [14, 15, 17, 18]. Although we expected some beneficial effects on microbiota composition, diversity and SCFA production, the microbiota analysis should be considered as explorative. Hence, it does not make sense to perform power analysis on selected bacteria groups post hoc. There is also no established method for calculating the power of a multivariate analysis, although some simulation-based approaches have been suggested. However, the fact that moderate changes in total microbiota $(1.5 \%)$ were observed with relatively low $p$ values $(<0.05)$ indicate that the sample size is sufficiently high.

Results from the FFQ assessment at baseline also showed that our participants slightly exceeded the criteria for allowed fibre intake (mean of $32.2 \mathrm{~g}$ per day). This indicates that the study population had higher habitual fibre intake than the general population with type 2 diabetes in Norway, and were on the other hand adherent to the Norwegian dietary recommendations of 25-35-g fibre per day [58]. This may have affected the baseline microbiota composition and diversity and thus its responsiveness to the prebiotic fibre. However, no significant correlation was found between baseline data such as fibre intake, microbial diversity or bifidobacteria levels on the bifidogenic response in the study. This is in contrast to other studies that reported more pronounced bifidogenic response with higher habitual fibre intake [19] and lower baseline levels of bifidobacteria $[14,15,59,60]$. Nevertheless, regarding the results from the FFQ, we cannot exclude a reporter bias due to the participants' knowledge of the nature of the study. All dietary assessment methods are known to be biased by both over- and underreporting. This is clearly illustrated by some of the extreme reported intakes of fibre in this study (Table 1). Hence, the data on dietary fibre intake should only be interpreted on group level and not individually.

\section{Conclusions}

In the present study, a daily supplement of inulin-type fructans induced a moderate, but significant increase in faecal levels of bifidobacteria, total SCFA, acetic acid and propionic acid in patients with type 2 diabetes. We were not able to detect any effects on the overall microbial diversity or faecal butyric acid. Our findings imply a moderate potential for these prebiotic fibres to improve the intestinal microenvironment in type 2 diabetes.

Acknowledgements Open Access funding provided by University of Oslo (incl Oslo University Hospital). The authors wish to thank research nurses Åse Halsne, Gøril Vinje and Gro Boezlijn at Oslo University Hospital and master students in clinical nutrition, University of Oslo: Oda K. S. Holm and Kristine D. Molven for help with data collection. We also thank Diabetes Laboratory, Central Laboratory, and Hormone Laboratory, Oslo University Hospital for blood samples and blood analysis. We thank Dimitrios Tzimorotas at Nofima for the microbiota analysis, and Gunn Helen Malmstrøm and Jennifer T. Fiennes at Unger-Vetlesen Institute, Lovisenberg Diaconal Hospital in Oslo for analysis of SCFA.

Author contributions EB: researched data, performed clinical assessments, and drafted the manuscript; SG and AA: initiated and designed the study and researched data; KIB: researched data; IR: performed microbiota analysis and data processing; JV: was responsible for laboratory analysis of faecal SCFA; IM: performed analysis of microbial data. All authors participated in manuscript writing, review, editing and discussion and have read and approved the final version.

Funding This research was supported by the DAM Foundation (Grant no. 2013-2-267) and the Norwegian Diabetes Association (Grant no. 36660), the Norwegian Levy on Agricultural Products (FFL; Project NFR 262300, 262306 and 262308) and Mills AS (Grant no. 36660), Oslo, Norway. Synergy 1 and placebo were provided by Beneo, Mannheim, Germany, which had no influence on study design nor interpretation of results.

Availability of data and material Data described in the manuscript and analytic code will be made available upon request pending application and approval.

\section{Compliance with ethical standards}

Conflict of interest The authors declare that they have no conflict of interest.

Ethical approval The trial was approved by the Regional Ethics Committee for Medical and Health Research and registered at clinicaltrials. gov (NCT02569684). The study was performed in accordance with the ethical standards laid down in the 1964 Declaration of Helsinki and its later amendments. Written informed consent was obtained from all patients prior to their inclusion in the study. 
Consent to participate Written informed consent was obtained from all patients prior to their inclusion in the study.

Consent for publication This manuscript is not being simultaneously submitted elsewhere and no portion of the data has been published elsewhere.

Open Access This article is licensed under a Creative Commons Attribution 4.0 International License, which permits use, sharing, adaptation, distribution and reproduction in any medium or format, as long as you give appropriate credit to the original author(s) and the source, provide a link to the Creative Commons licence, and indicate if changes were made. The images or other third party material in this article are included in the article's Creative Commons licence, unless indicated otherwise in a credit line to the material. If material is not included in the article's Creative Commons licence and your intended use is not permitted by statutory regulation or exceeds the permitted use, you will need to obtain permission directly from the copyright holder. To view a copy of this licence, visit http://creativecommons.org/licenses/by/4.0/.

\section{References}

1. American Diabetes Association (2019) 5. Lifestyle management: standards of medical care in diabetes-2019. Diabetes Care 42(Suppl 1):S46-S60. https://doi.org/10.2337/dc19-S005

2. Silva FM, Kramer CK, de Almeida JC, Steemburgo T, Gross JL, Azevedo MJ (2013) Fiber intake and glycemic control in patients with type 2 diabetes mellitus: a systematic review with meta-analysis of randomized controlled trials. Nutr Rev 71(12):790-801. https://doi.org/10.1111/nure.12076

3. Reynolds A, Mann J, Cummings J, Winter N, Mete E, Te Morenga L (2019) Carbohydrate quality and human health: a series of systematic reviews and meta-analyses. Lancet 393(10170):434-445. https://doi.org/10.1016/s0140-6736(18)31809-9

4. Gibson GR, Hutkins R, Sanders ME, Prescott SL, Reimer RA, Salminen SJ, Scott K, Stanton C, Swanson KS, Cani PD, Verbeke K, Reid G (2017) Expert consensus document: The International Scientific Association for Probiotics and Prebiotics (ISAPP) consensus statement on the definition and scope of prebiotics. Nat Rev Gastroenterol Hepatol 14(8):491-502. https://doi. org/10.1038/nrgastro.2017.75

5. O'Callaghan A, van Sinderen D (2016) Bifidobacteria and their role as members of the human gut microbiota. Front Microbiol 7:925. https://doi.org/10.3389/fmicb.2016.00925

6. Correa-Oliveira R, Fachi JL, Vieira A, Sato FT, Vinolo MA (2016) Regulation of immune cell function by short-chain fatty acids. Clin Transl Immunol 5(4):e73. https://doi.org/10.1038/ cti.2016.17

7. Cani PD, Everard A, Duparc T (2013) Gut microbiota, enteroendocrine functions and metabolism. Curr Opin Pharmacol 13(6):935-940. https://doi.org/10.1016/j.coph.2013.09.008

8. Gao Z, Yin J, Zhang J, Ward RE, Martin RJ, Lefevre M, Cefalu WT, Ye J (2009) Butyrate improves insulin sensitivity and increases energy expenditure in mice. Diabetes 58(7):1509-1517. https://doi.org/10.2337/db08-1637

9. Lin HV, Frassetto A, Kowalik EJ Jr, Nawrocki AR, Lu MM, Kosinski JR, Hubert JA, Szeto D, Yao X, Forrest G, Marsh DJ (2012) Butyrate and propionate protect against diet-induced obesity and regulate gut hormones via free fatty acid receptor 3-independent mechanisms. PLoS ONE 7(4):e35240. https://doi. org/10.1371/journal.pone.0035240
10. Hamer HM, Jonkers D, Venema K, Vanhoutvin S, Troost FJ, Brummer RJ (2008) Review article: the role of butyrate on colonic function. Aliment Pharmacol Ther 27(2):104-119. https://doi.org /10.1111/j.1365-2036.2007.03562.x

11. Forslund K, Hildebrand F, Nielsen T, Falony G, Le Chatelier E, Sunagawa S, Prifti E, Vieira-Silva S, Gudmundsdottir V, Pedersen HK, Arumugam M, Kristiansen K, Voigt AY, Vestergaard H, Hercog R, Costea PI, Kultima JR, Li J, Jorgensen T, Levenez F, Dore J, Nielsen HB, Brunak S, Raes J, Hansen T, Wang J, Ehrlich SD, Bork P, Pedersen O (2015) Disentangling type 2 diabetes and metformin treatment signatures in the human gut microbiota. Nature 528(7581):262-266. https://doi.org/10.1038/nature15766

12. Karlsson FH, Tremaroli V, Nookaew I, Bergstrom G, Behre CJ, Fagerberg B, Nielsen J, Backhed F (2013) Gut metagenome in European women with normal, impaired and diabetic glucose control. Nature 498(7452):99-103. https://doi.org/10.1038/natur e12198

13. Vrieze A, Van Nood E, Holleman F, Salojarvi J, Kootte RS, Bartelsman JF, Dallinga-Thie GM, Ackermans MT, Serlie MJ, Oozeer R, Derrien M, Druesne A, Van Hylckama Vlieg JE, Bloks VW, Groen AK, Heilig HG, Zoetendal EG, Stroes ES, de Vos WM, Hoekstra JB, Nieuwdorp M (2012) Transfer of intestinal microbiota from lean donors increases insulin sensitivity in individuals with metabolic syndrome. Gastroenterology 143(4):913-916. https://doi.org/10.1053/j.gastro.2012.06.031

14. Kolida S, Meyer D, Gibson GR (2007) A double-blind placebocontrolled study to establish the bifidogenic dose of inulin in healthy humans. Eur J Clin Nutr 61(10):1189-1195. https://doi. org/10.1038/sj.ejcn.1602636

15. Bouhnik Y, Raskine L, Simoneau G, Vicaut E, Neut C, Flourie B, Brouns F, Bornet FR (2004) The capacity of nondigestible carbohydrates to stimulate fecal bifidobacteria in healthy humans: a double-blind, randomized, placebo-controlled, parallel-group, dose-response relation study. Am J Clin Nutr 80(6):1658-1664

16. Ten Bruggencate SJ, Bovee-Oudenhoven IM, Lettink-Wissink ML, Katan MB, van der Meer R (2006) Dietary fructooligosaccharides affect intestinal barrier function in healthy men. J Nutr 136(1):70-74. https://doi.org/10.1093/jn/136.1.70

17. Lomax AR, Cheung LV, Tuohy KM, Noakes PS, Miles EA, Calder PC (2012) Beta2-1 fructans have a bifidogenic effect in healthy middle-aged human subjects but do not alter immune responses examined in the absence of an in vivo immune challenge: results from a randomised controlled trial. Br J Nutr 108(10):1818-1828. https://doi.org/10.1017/s0007114511007276

18. Salazar N, Dewulf EM, Neyrinck AM, Bindels LB, Cani PD, Mahillon J, de Vos WM, Thissen JP, Gueimonde M, de Los Reyes-Gavilan CG, Delzenne NM (2014) Inulin-type fructans modulate intestinal Bifidobacterium species populations and decrease fecal short-chain fatty acids in obese women. Clin Nutr. https://doi.org/10.1016/j.clnu.2014.06.001

19. Healey G, Murphy R, Butts C, Brough L, Whelan K, Coad J (2018) Habitual dietary fibre intake influences gut microbiota response to an inulin-type fructan prebiotic: a randomised, double-blind, placebo-controlled, cross-over, human intervention study. Br J Nutr 119(2):176-189. https://doi.org/10.1017/s0007 114517003440

20. Baxter NT, Schmidt AW, Venkataraman A, Kim KS, Waldron C, Schmidt TM (2019) Dynamics of human gut microbiota and shortchain fatty acids in response to dietary interventions with three fermentable fibers. MBio. https://doi.org/10.1128/mBio.02566-18

21. Liu F, Li P, Chen M, Luo Y, Prabhakar M, Zheng H, He Y, Qi Q, Long H, Zhang Y, Sheng H, Zhou H (2017) Fructooligosaccharide (FOS) and galactooligosaccharide (GOS) increase Bifidobacterium but reduce butyrate producing bacteria with adverse glycemic metabolism in healthy young population. Sci Rep 7(1):11789. https://doi.org/10.1038/s41598-017-10722-2 
22. Vandeputte D, Falony G, Vieira-Silva S, Wang J, Sailer M, Theis S, Verbeke K, Raes J (2017) Prebiotic inulin-type fructans induce specific changes in the human gut microbiota. Gut 66(11):1968-1974. https://doi.org/10.1136/gutjnl-2016-313271

23. Holscher HD, Bauer LL, Gourineni V, Pelkman CL, Fahey GC Jr, Swanson KS (2015) Agave inulin supplementation affects the fecal microbiota of healthy adults participating in a randomized, double-blind, placebo-controlled, crossover trial. J Nutr 145(9):2025-2032. https://doi.org/10.3945/jn.115.217331

24. Ramirez-Farias C, Slezak K, Fuller Z, Duncan A, Holtrop G, Louis P (2009) Effect of inulin on the human gut microbiota: stimulation of Bifidobacterium adolescentis and Faecalibacterium prausnitzii. Br J Nutr 101(4):541-550. https://doi. org/10.1017/s0007114508019880

25. Gurung M, Li Z, You H, Rodrigues R, Jump DB, Morgun A, Shulzhenko N (2020) Role of gut microbiota in type 2 diabetes pathophysiology. EBioMedicine 51:102590. https://doi. org/10.1016/j.ebiom.2019.11.051

26. Mahboobi S, Rahimi F, Jafarnejad S (2018) Effects of prebiotic and synbiotic supplementation on glycaemia and lipid profile in type 2 diabetes: a meta-analysis of randomized controlled trials. Adv Pharm Bull 8(4):565-574. https://doi.org/10.15171 /apb.2018.065

27. Bouhnik Y, Vahedi K, Achour L, Attar A, Salfati J, Pochart P, Marteau P, Flourie B, Bornet F, Rambaud JC (1999) Shortchain fructo-oligosaccharide administration dose-dependently increases fecal bifidobacteria in healthy humans. J Nutr 129(1):113-116

28. Cani PD, Lecourt E, Dewulf EM, Sohet FM, Pachikian BD, Naslain D, De Backer F, Neyrinck AM, Delzenne NM (2009) Gut microbiota fermentation of prebiotics increases satietogenic and incretin gut peptide production with consequences for appetite sensation and glucose response after a meal. Am J Clin Nutr 90(5):1236-1243. https://doi.org/10.3945/ajcn.2009.28095

29. Carlsen MH, Lillegaard IT, Karlsen A, Blomhoff R, Drevon CA, Andersen LF (2010) Evaluation of energy and dietary intake estimates from a food frequency questionnaire using independent energy expenditure measurement and weighed food records. Nutr J 9:37. https://doi.org/10.1186/1475-2891-9-37

30. Carlsen MH, Karlsen A, Lillegaard IT, Gran JM, Drevon CA, Blomhoff R, Andersen LF (2011) Relative validity of fruit and vegetable intake estimated from an FFQ, using carotenoid and flavonoid biomarkers and the method of triads. Br J Nutr 105(10):1530-1538. https://doi.org/10.1017/s0007114510005246

31. Moen B, Henjum K, Mage I, Knutsen SH, Rud I, Hetland RB, Paulsen JE (2016) Effect of dietary fibers on cecal microbiota and intestinal tumorigenesis in azoxymethane treated A/J Min/+ mice. PLoS ONE 11(5):e0155402. https://doi.org/10.1371/journ al.pone. 0155402

32. Caporaso JG, Lauber CL, Walters WA, Berg-Lyons D, Huntley J, Fierer N, Owens SM, Betley J, Fraser L, Bauer M, Gormley N, Gilbert JA, Smith G, Knight R (2012) Ultra-high-throughput microbial community analysis on the Illumina HiSeq and MiSeq platforms. ISME J 6(8):1621-1624. https://doi.org/10.1038/ismej .2012 .8

33. Walters W, Hyde ER, Berg-Lyons D, Ackermann G, Humphrey G, Parada A, Gilbert JA, Jansson JK, Caporaso JG, Fuhrman JA, Apprill A, Knight R (2016) Improved bacterial 16S rRNA Gene (V4 and V4-5) and fungal internal transcribed spacer marker gene primers for microbial community surveys. mSystems. https://doi. org/10.1128/mSystems.00009-15

34. Parada AE, Needham DM, Fuhrman JA (2016) Every base matters: assessing small subunit rRNA primers for marine microbiomes with mock communities, time series and global field samples. Environ Microbiol 18(5):1403-1414. https://doi. org/10.1111/1462-2920.13023
35. Apprill A, McNally S, Parsons R, Weber L (2015) Minor revision to V4 region SSU rRNA 806R gene primer greatly increases detection of SAR11 bacterioplankton. Aquat Microb Ecol 75:129_ 137. https://doi.org/10.3354/ame01753

36. Caporaso JG, Kuczynski J, Stombaugh J, Bittinger K, Bushman FD, Costello EK, Fierer N, Pena AG, Goodrich JK, Gordon JI, Huttley GA, Kelley ST, Knights D, Koenig JE, Ley RE, Lozupone CA, McDonald D, Muegge BD, Pirrung M, Reeder J, Sevinsky JR, Turnbaugh PJ, Walters WA, Widmann J, Yatsunenko T, Zaneveld J, Knight R (2010) QIIME allows analysis of high-throughput community sequencing data. Nat Methods 7(5):335-336. https ://doi.org/10.1038/nmeth.f.303

37. Zijlstra JB, Beukema J, Wolthers BG, Byrne BM, Groen A, Dankert J (1977) Pretreatment methods prior to gaschromatographic analysis of volatile fatty acids from faecal samples. Clin Chim Acta 78(2):243-250

38. Hoverstad T, Fausa O, Bjorneklett A, Bohmer T (1984) Shortchain fatty acids in the normal human feces. Scand J Gastroenterol 19(3):375-381

39. Inagaki N, Onouchi H, Maezawa H, Kuroda S, Kaku K (2015) Once-weekly trelagliptin versus daily alogliptin in Japanese patients with type 2 diabetes: a randomised, double-blind, phase 3 , non-inferiority study. Lancet Diabetes Endocrinol 3(3):191-197. https://doi.org/10.1016/s2213-8587(14)70251-7

40. Jansen JJ, Hoefsloot HCJ, van der Greef J, Timmerman ME, Westerhuis JA, Smilde AK (2005) ASCA: analysis of multivariate data obtained from an experimental design. J Chemometr 19(9):469481. https://doi.org/10.1002/cem.952

41. Eriksson L, Byrne T, Johansson E, Trygg J, Vikström C (2013) Multi- and megavariate data analysis: basic principles and applications

42. Wilson B, Whelan K (2017) Prebiotic inulin-type fructans and galacto-oligosaccharides: definition, specificity, function, and application in gastrointestinal disorders. J Gastroenterol Hepatol 32(Suppl 1):64-68. https://doi.org/10.1111/jgh.13700

43. Ghoddusi HB, Grandison MA, Grandison AS, Tuohy KM (2007) In vitro study on gas generation and prebiotic effects of some carbohydrates and their mixtures. Anaerobe 13(5-6):193-199. https ://doi.org/10.1016/j.anaerobe.2007.06.002

44. Sanders ME, Lenoir-Wijnkoop I, Salminen S, Merenstein DJ, Gibson GR, Petschow BW, Nieuwdorp M, Tancredi DJ, Cifelli CJ, Jacques P, Pot B (2014) Probiotics and prebiotics: prospects for public health and nutritional recommendations. Ann N Y Acad Sci 1309(1):19-29. https://doi.org/10.1111/nyas.12377

45. Cani PD, Neyrinck AM, Fava F, Knauf C, Burcelin RG, Tuohy KM, Gibson GR, Delzenne NM (2007) Selective increases of bifidobacteria in gut microflora improve high-fat-diet-induced diabetes in mice through a mechanism associated with endotoxaemia. Diabetologia 50(11):2374-2383. https://doi.org/10.1007/s0012 5-007-0791-0

46. Cronin M, Ventura M, Fitzgerald GF, van Sinderen D (2011) Progress in genomics, metabolism and biotechnology of bifidobacteria. Int J Food Microbiol 149(1):4-18. https://doi.org/10.1016/j. ijfoodmicro.2011.01.019

47. Tandon D, Haque MM, Gote M, Jain M, Bhaduri A, Dubey AK, Mande SS (2019) A prospective randomized, double-blind, placebo-controlled, dose-response relationship study to investigate efficacy of fructo-oligosaccharides (FOS) on human gut microflora. Sci Rep 9(1):5473. https://doi.org/10.1038/s41598-01941837-3

48. Parnell JA, Reimer RA (2009) Weight loss during oligofructose supplementation is associated with decreased ghrelin and increased peptide YY in overweight and obese adults. Am J Clin Nutr 89(6):1751-1759. https://doi.org/10.3945/ajcn.2009.27465

49. Genta S, Cabrera W, Habib N, Pons J, Carillo IM, Grau A, Sanchez S (2009) Yacon syrup: beneficial effects on obesity and 
insulin resistance in humans. Clin Nutr (Edinburgh, Scotland) 28(2):182-187. https://doi.org/10.1016/j.clnu.2009.01.013

50. Morrison DJ, Preston T (2016) Formation of short chain fatty acids by the gut microbiota and their impact on human metabolism. Gut Microbes 7(3):189-200. https://doi.org/10.1080/19490 976.2015.1134082

51. Scheppach W, Weiler F (2004) The butyrate story: old wine in new bottles? Curr Opin Clin Nutr Metab Care 7(5):563-567

52. Moens F, Weckx S, De Vuyst L (2016) Bifidobacterial inulin-type fructan degradation capacity determines cross-feeding interactions between bifidobacteria and Faecalibacterium prausnitzii. Int $\mathbf{J}$ Food Microbiol 231:76-85. https://doi.org/10.1016/j.ijfoodmicr o.2016.05.015

53. Rios-Covian D, Gueimonde M, Duncan SH, Flint HJ, de los Reyes-Gavilan CG (2015) Enhanced butyrate formation by crossfeeding between Faecalibacterium prausnitzii and Bifidobacterium adolescentis. FEMS Microbiol Lett 362(21):78. https://doi. org/10.1093/femsle/fnv176

54. Rossi M, Corradini C, Amaretti A, Nicolini M, Pompei A, Zanoni S, Matteuzzi D (2005) Fermentation of fructooligosaccharides and inulin by bifidobacteria: a comparative study of pure and fecal cultures. Appl Environ Microbiol 71(10):6150-6158. https://doi. org/10.1128/aem.71.10.6150-6158.2005

55. Falony G, Lazidou K, Verschaeren A, Weckx S, Maes D, De Vuyst L (2009) In vitro kinetic analysis of fermentation of prebiotic inulin-type fructans by Bifidobacterium species reveals four different phenotypes. Appl Environ Microbiol 75(2):454-461. https://doi. org/10.1128/aem.01488-08

56. Sonnenburg ED, Zheng H, Joglekar P, Higginbottom SK, Firbank SJ, Bolam DN, Sonnenburg JL (2010) Specificity of polysaccharide use in intestinal bacteroides species determines dietinduced microbiota alterations. Cell 141(7):1241-1252. https:// doi.org/10.1016/j.cell.2010.05.005

57. David LA, Maurice CF, Carmody RN, Gootenberg DB, Button JE, Wolfe BE, Ling AV, Devlin AS, Varma Y, Fischbach MA, Biddinger SB, Dutton RJ, Turnbaugh PJ (2014) Diet rapidly and reproducibly alters the human gut microbiome. Nature 505(7484):559-563. https://doi.org/10.1038/nature 12820

58. Aas A-M, Johansson L, Bjerkan K, Lorentsen N, Mostad I (2013) Do Norwegians with diabetes have a healthier diet than the general population? Norsk Epidemiol 23:61-74. https://doi.org/10.5324/ nje.v23i1.1604

59. Tuohy KM, Kolida S, Lustenberger AM, Gibson GR (2001) The prebiotic effects of biscuits containing partially hydrolysed guar gum and fructo-oligosaccharides-a human volunteer study. Br J Nutr 86(3):341-348

60. de Preter V, Vanhoutte T, Huys G, Swings J, Rutgeerts P, Verbeke K (2008) Baseline microbiota activity and initial bifidobacteria counts influence responses to prebiotic dosing in healthy subjects. Aliment Pharmacol Ther 27(6):504-513. https://doi.org/10.111 1/j.1365-2036.2007.03588.x 